\section{Role of superhydrophobicity in the biological activity of fibronectin at the cell- material interface $\dagger$}

\author{
José Ballester-Beltrán, $\ddagger^{a}$ Patricia Rico, $\dagger^{a b}$ David Moratal, ${ }^{a}$ Wenlong Song, ${ }^{c d}$ João F. Mano ${ }^{c d}$ \\ and Manuel Salmerón-Sánchez ${ }^{* a b e}$
}

Received 13th June 2011, Accepted 24th August 2011

DOI: $10.1039 / \mathrm{c} 1 \mathrm{sm} 06102 \mathrm{j}$

\begin{abstract}
Protein adsorption and cellular behavior depend strongly on the wettability of substrates. Such studies are scarce for surfaces exhibiting extreme values of contact angles. Fibronectin (FN) adsorption and adhesion of MC3T3-E1 cells were investigated on superhydrophobic polystyrene (SH-PS) surfaces and compared with the corresponding smooth polystyrene (PS) substrate and the control glass. The FN surface density was lower on the SH-PS than on PS, and the adsorbed protein showed altered conformation of cell adhesion domains, as obtained by ELISA with monoclonal antibodies. Cell adhesion occurred on the SH-PS without the formation of mature focal adhesions, as assessed by immunofluorescence for vinculin, talin and paxillin. Correspondingly, the development of the actin cytoskeleton was delayed and without the presence of defined F-actin fibers. FAK phosphorylation was reduced on SH-PS, as compared with PS and the control glass. Also, cell contractility was diminished on the SH-PS as revealed by phosphorylation of myosin light chain (pMLC). Likewise, FN reorganization and secretion were impaired on the superhydrophobic surfaces. Cell proliferation was significantly lower in SH-PS as compared with PS up to 21 days of culture.
\end{abstract}

\section{Introduction}

The fact that protein adsorption and cell adhesion depend on the physical and chemical properties of the material surface is well established in the literature. ${ }^{1-3}$ Even though wettability is a rough-macroscopic parameter that reflects surface chemistry and micro/nanotopography, it is discussed in the literature as one of the most important parameters modulating cell-material interactions. ${ }^{4,5}$ The confusion is justified as most of these studies have been carried out using smooth surfaces ranging within the standard hydrophilic and hydrophobic regimes (i.e. WCA

${ }^{a}$ Center for Biomaterials and Tissue Engineering, Universitat Politècnica de València, Spain. E-mail: cbiomat@upvnet.upv.es; Fax: +34 963877276; Tel: +34 963888930; masalsan@fis.upv.es; +34 963877276; +34 963877275

${ }^{b} C I B E R$ de Bioingeniería, Biomateriales y Nanomedicina (CIBER-BBN), Valencia, Spain

'3B's Research Group-Biomaterials, Biodegradables and Biomimetics, Headquarters of the European Institute of Excellence on Tissue Engineering and Regenerative Medicine, Department of Polymer Engineering, University of Minho, Guimarães, Portugal. E-mail: info@, 3bs.uminho.pt; Fax: +351 253510900; Tel: +351 253510900

${ }^{d} I B B$ - Institute for Biotechnology and Bioengineering, PT Associated Laboratory, Guimarães, Portugal

${ }^{e}$ Regenerative Medicine Unit, Centro de Investigación Príncipe Felipe, Valencia, Spain

$\dagger$ Electronic supplementary information (ESI) available. See DOI: $10.1039 / \mathrm{c} 1 \mathrm{sm} 06102 \mathrm{j}$

\$ These two authors contributed equally to this work. between 30 and $100^{\circ}$ ), even if very different chemistries can provide similar wettability but different cell behavior afterwards. ${ }^{6}$ Highly water repellent surfaces could provide new insights on how proteins and cells react in more extreme conditions of wettability. Superhydrophobic surfaces have generated an increasing interest in the past few years owing to their industrial and scientific applications. ${ }^{7-11}$ Nevertheless, the use of this kind of materials for biomedical applications has been poorly studied. ${ }^{12-15}$ Superhydrophobic surfaces have been proposed for anti-bioadhesion applications, seeking to prevent protein adsorption and cell adhesion, such as blood compatible materials ${ }^{16}$ coating the internal surface of artificial organ implantations, manmade blood vessels and other medical devices in contact with blood. ${ }^{17,18}$ Nonetheless, only a few authors have studied cell response to such extreme characteristics. ${ }^{19-22}$

Living cells cannot interact directly with synthetic materials, but they readily attach to the extracellular matrix (ECM) proteins adsorbed on the substrate coming from physiological fluids in vivo or culture medium in vitro. ${ }^{23}$ The adsorbed matrix proteins are recognized by integrins, cell surface receptors that provide trans-membrane links between the ECM and the actin cytoskeleton. ${ }^{24}$ Afterwards, integrins cluster and develop focal adhesion complexes that anchorage cells to the material surface. Fibronectin (FN) coated surfaces allow integrin-mediated cell adhesion, mainly through the $\alpha_{5} \beta_{1}$ integrin, ${ }^{25}$ which clusters near the periphery of cells and direct the subsequent cellular response triggered by phosphorylation of focal adhesion kinases (FAKs). 
Apart from adhesion on FN-coated materials, cells also secrete and reorganize this protein at the material interface into a specific fibrillar pattern, ${ }^{26-29}$ as an attempt to form a matrix on the material surface. ${ }^{30}$ Thus, cells organize their environment continuously by matrix reorganization and secretion.

Our aim was to investigate the role of surfaces with extreme wettability properties at the interface with biological media to understand interactions with ECM proteins and cell responses and to gain further insights before the intended applications of these surfaces. ${ }^{16-18}$ Polystyrene is a widely used polymer to produce tissue culture ware. This work addresses the study of FN adsorption and cell response on superhydrophobic polystyrene as obtained from smooth polystyrene by a phase-separation process. Whilst having the same surface chemistry, the superhydrophobic surfaces differ in wettability from the smooth one as a consequence of the particular surface nano/microstructure. ${ }^{31}$

\section{Experimental}

\section{Materials}

Many techniques have been proposed to produce artificial superhydrophobic surfaces, including phase separation, template synthesis, electrochemical deposition, electrohydrodynamics, or crystallization control. ${ }^{32}$ Among them, simple, economical and one-step procedures to produce superhydrophobic surfaces have been developed. For example, Erbil et al. reported a simple method for forming superhydrophobic surfaces in polypropylene by combining solvents and adequate drying temperatures. ${ }^{33} \mathrm{~A}$ phase inversion-based methodology was also developed involving the use of a solvent and non-solvent to develop surface features giving rise to superhydrophobic characteristics in biodegradable polymers. ${ }^{34}$ Polystyrene films were also processed using such methodology to produce superhydrophobic surfaces, ${ }^{19}$ and the response to different cell types was investigated. ${ }^{35}$ A similar methodology was employed in this work to process the superhydrophobic substrates. Briefly, a $70 \mathrm{mg} \mathrm{mL}^{-1}$ solution of PS (injection molding grade) in tetrahydrofuran was prepared, and $0.65 \mathrm{~mL}$ of ethanol was added per each $\mathrm{mL}$ of the PS solution. A few drops of this mixture were dipped onto a clean PS smooth surface prepared by compression molding. After $10 \mathrm{~s}$ on air, the substrate with the mixture was immersed in ethanol for $1 \mathrm{~min}$. Afterwards, the surface was dried under a nitrogen flow.

Glass coverslips were used as control surfaces. Glass coverslips were cleaned with $70 \% \mathrm{H}_{2} \mathrm{SO}_{4}$ and $30 \% \mathrm{H}_{2} \mathrm{O}_{2}$ at room temperature for $1 \mathrm{~h}$, rinsed with deionized $\mathrm{H}_{2} \mathrm{O}$, rinsed with $95 \%$ ethanol, and dried under a stream of nitrogen.

Surface wettability was assessed by water contact angle (WCA) measurements using a DATAPHYSICS-OCA20 instrument and the sessile drop method.

\section{X-Ray photoelectron spectroscopy (XPS)}

The XPS experiments were performed in a PHI 5500 Multitechnique System (from Physical Electronics) with a monochromatic X-ray source and calibrated using the 3d5/2 line of $\mathrm{Ag}$. The analyzed area was a circle of $0.8 \mathrm{~mm}$ diameter, and the selected resolution for the spectra was $23.5 \mathrm{eV}$ of pass energy and $0.1 \mathrm{eV}$ per step. All measurements were made in an ultra high vacuum (UHV) chamber pressure. The XPS elemental sensitivity factors according to the MULTIPAK program for PHI instruments were used.

\section{Atomic force microscopy (AFM)}

AFM was performed in a NanoScope III from Digital Instruments (Santa Barbara, CA) operating in the tapping mode; the Nanoscope 5.30r2 software version was used. Si-cantilevers from Veeco (Manchester, UK) were used with a force constant of $2.8 \mathrm{~N} \mathrm{~m}^{-1}$ and a resonance frequency of $75 \mathrm{kHz}$. The phase signal was set to zero at a frequency $5-10 \%$ lower than the resonance one. The drive amplitude was $200 \mathrm{mV}$ and the amplitude setpoint (Asp) was $1.4 \mathrm{~V}$. The ratio between the amplitude setpoint and the free amplitude (Asp/A0) was kept equal to 0.7.

\section{Fibronectin adsorption}

Sample disks were coated with FN $20 \mu \mathrm{g} \mathrm{mL}^{-1}$ (Sigma-Aldrich) in Dulbecco's phosphate buffered saline (DPBS, Invitrogen) for 1 hour at room temperature. The amount of adsorbed FN was quantified by image analysis of the western blot as explained elsewhere. $^{36}$

Two antibodies were used as structural probes for adsorbed $\mathrm{FN}$ in an enzyme-linked immunosorbent assay (ELISA). Substrates were incubated in FN dilution $\left(20 \mu \mathrm{g} \mathrm{mL}^{-1}\right)$ for 1 hour at room temperature. Afterwards surfaces were blocked against nonspecific antibody binding using blocking buffer (DPBS/1\% BSA) for $30 \mathrm{~min}$ at room temperature. Substrates were then incubated in primary antibody (1: 4000 for HFN7.1 (Developmental Hybridoma) and 1: 1000 for mAb1937 (R\&D Systems)) in blocking buffer for 1 hour at $37^{\circ} \mathrm{C}$. After several washings with DPBS $/ 0.5 \%$ Tween 20, substrates were incubated in $1: 5000$ alkaline phosphatase conjugated antibody (Jackson Immunoresearch) for 1 hour at $37^{\circ} \mathrm{C}$, washed and incubated in 4-methylumbelliferyl phosphate for $45 \mathrm{~min}$ at $37^{\circ} \mathrm{C}$. Reaction products were quantified using a Victor III (Perkin Elmer Group) at $360 \mathrm{~nm}$ excitation/465 $\mathrm{nm}$ emission.

\section{Cell culture}

MC3T3-E1 cells were obtained from RIKEN Cell Bank (Japan). Cells were maintained in DMEM medium supplemented with $10 \%$ fetal bovine serum (Fisher) and 1\% penicillin-streptomycin (Lonza) and passaged twice a week using standard techniques.

Prior to seeding on substrates, sample disks were coated with FN $20 \mu \mathrm{g} \mathrm{mL}^{-1}$ for 1 hour at room temperature. Then, 3000 cells $\mathrm{cm}^{-2}$ were placed onto each substrate in serum-free conditions and were maintained at $37^{\circ} \mathrm{C}$ in a humidified atmosphere under $5 \% \mathrm{CO}_{2}$. Each experiment was performed in triplicate.

\section{Cell adhesion}

In rigor, adhesion precedes spreading and is the simple physical attachment of a cell through integrins and other receptors to a surface. ${ }^{11}$ Since we are looking at focal adhesions and actin stress fibers, which can only occur during or after a cell has spread, it would be more appropriate to use the term spreading rather than adhesion but still we have maintained the second one throughout the manuscript. Cell adhesion was studied by 
immunodetection of vinculin, talin and paxillin proteins. After 3 hours of culture cells were washed in DPBS and fixed in 10\% formalin solution (Sigma) at $4{ }^{\circ} \mathrm{C}$ for 1 hour. Samples were then rinsed and permeabilized (saccharose $0.3 \mathrm{M}, \mathrm{NaCl}$ (Sigma) $50 \mathrm{mM}, \mathrm{MgCl}_{2}$ hexahydrate (Scharlab) $3 \mathrm{mM}$, Hepes (Sigma) $20 \mathrm{mM}$ and Triton X-100 (Sigma) $0.5 \%$ ) for 5 minutes and washed with DPBS. Then, samples were incubated in DPBS $/ 1 \%$ BSA in order to reduce the background signal. Cells were then incubated with either anti-vinculin antibody (Sigma) diluted $1: 400$, anti-talin antibody (Sigma) diluted $1: 400$ or anti-paxillin antibody (Sigma) diluted 1:200 for 1 hour. The samples were then rinsed in DPBS $/ 0.5 \%$ Tween 20 , followed by incubation with $\mathrm{Cy} 3$ conjugated secondary antibody (Jackson Immunoresearch) diluted $1: 200$ and BODIPY FL phallacidin (Molecular probes) diluted 1/40. Finally the samples were washed before being mounted in Vectashield containing 4',6-diamidino-2-phenylindole (DAPI, Atom). A confocal microscopy (Leica TCS SP2 AOBS) was used.

\section{Cell contractility}

Cells were cultured on FN-coated materials for $3 \mathrm{~h}$ in serum-free conditions and immunostained for phosphorylated myosin light chain (pMLC). Briefly, cultures were fixed in 3.7\% formaldehyde in PBS for $15 \mathrm{~min}$ at room temperature and then blocked in blocking buffer ( $5 \%$ goat serum and $0.3 \%$ Triton X-100 in DPBS) for $1 \mathrm{~h}$. Samples were sequentially incubated in pMLC rabbit antibody (1:200, Cell Signalling) and goat anti-rabbit Cy3 conjugated secondary antibody $(1: 100)$. Samples were washed before being mounted in Vectashield containing DAPI.

\section{Cell signaling}

After 3 hours of culture, cells were lysed with RIPA buffer (Tris$\mathrm{HCl} 50 \mathrm{mM}, 1 \%$ Nonidet P-40, 0.25\% Na deoxycholate, $\mathrm{NaCl}$ $150 \mathrm{mM}$, EDTA $1 \mathrm{mM}$ ) supplemented with protease inhibitor cocktail tablets (Complete, Roche). Proteins were concentrated using Microcon YM-30 Centrifugal Filter devices (Millipore) as the manufacturer described.

To determine FAK protein expression and its phosphorylated form ( $\mathrm{pFAK}$ ), concentrated samples were subjected to 7\% SDSPAGE gel electrophoresis. Proteins were transferred to a positively charged PVDF membrane (GE Healthcare) using a semidry transfer cell system (Biorad) and blocked by immersion in 5\% skimmed milk in PBS for $30 \mathrm{~min}$ at room temperature. The blot was incubated with anti-FAK antibody (Upstate) and antipFAK antibody (Millipore) diluted 1:2500 in PBS containing $0.1 \%$ Tween 20 and $2 \%$ skimmed milk. After several washes with PBS $/ 0.1 \%$ Tween 20, the blot was incubated in horseradish peroxidase-conjugated antibody (GE Healthcare) diluted 1 : 50000 in PBS containing 0.1\% Tween 20 and 2\% milk for 1 hour at room temperature. After several washes with PBS containing $0.1 \%$ Tween and $2 \%$ milk, immunoreactive bands were visualized using the Supersignal West-femto Maximum Sensitivity Substrate (Thermo Scientific).

\section{Scanning electron microscopy}

After 1 day of culture, cells were visualized using scanning electron microscopy (SEM). Cells were fixed in $2.5 \%$ glutaraldehyde
(Panreac) for 1 hour at $4{ }^{\circ} \mathrm{C}$ and after several washes in DPBS, samples were dehydrated using graded ethanol solutions $(30,50$, $70,80,90,96,100$ and $100 \%$ ) and finally coated with gold prior to SEM observations (JEOL JSM-6300).

\section{Fibronectin reorganization}

FN reorganization was studied at 4 different time points. Samples were coated with FN $\left(20 \mu \mathrm{g} \mathrm{mL}^{-1}\right)$ and seeded with MC3T3-E1 (3000 cells $\mathrm{cm}^{-2}$ ). Once the culture was finished (2.5 hours, 5 hours, 1 day or 3 days), cells were fixed with $10 \%$ formalin for 1 hour at $4{ }^{\circ} \mathrm{C}$. Then samples were washed with DPBS and cells were permeabilized for $5 \mathrm{~min}$ at room temperature. Afterwards, samples were blocked in DPBS/1\% BSA and incubated with anti-FN (Sigma), 1:400 in blocking buffer for 1 hour at room temperature. Samples were then rinsed in DPBS $/ 0.5 \%$ Tween 20, and incubated in Cy3-conjugated secondary antibody (Jackson Immunoresearch, 1:400) and BODIPY FL phallacidin (Molecular probes, 1:40). Finally, samples were washed before being mounted in Vectashield containing DAPI (Atom). A confocal microscope (Leica TCS SP2 AOBS) was used.

\section{Image processing}

All image processing and analysis were done using an in-house software developed under MATLAB R2006a (The MathWorks, Inc., Natick, MA).

\section{Statistical analysis}

Results are shown as average \pm standard deviation. Protein quantification and WCA changes were analyzed by Student's $t$ model and conformational results by ANOVA $(p<0.05$ was considered significant and indicated with a symbol on each figure).

\section{Results and discussion}

\section{Fibronectin adsorption on superhydrophobic PS}

Superhydrophobic polystyrene (SH-PS) was prepared using a phase separation methodology that leads to precipitation of PS on the surface and the formation of a rough surface on the original PS one (Fig. 1A). ${ }^{21}$ The surface roughness of PS and SH-PS was measured by AFM and displayed in Table 1. This treatment transforms the original hydrophobic PS surface into a superhydrophobic one, as the measured water contact angles (WCAs) confirms (Fig. 1B). Glass is included as a well-known control substrate for protein adsorption and cell adhesion.

The composition of the surface was investigated by XPS. Fig. 1C shows similar C 1s spectra for both surfaces, with different intensities as a consequence of the surface roughness. As expected, the $\mathrm{O} 1 \mathrm{~s}$ spectra did not show any significant peak for either PS or SH-PS. That is to say, surface chemistry is maintained on the SH-PS and superhydrophobicity is a direct consequence of the roughness of the surface (Table 1). Cassie and Baxter postulated that the surface roughness leads to superhydrophobicity as a consequence of the fact that the liquid does not intrude into the valleys of the rough surface and a fraction of 

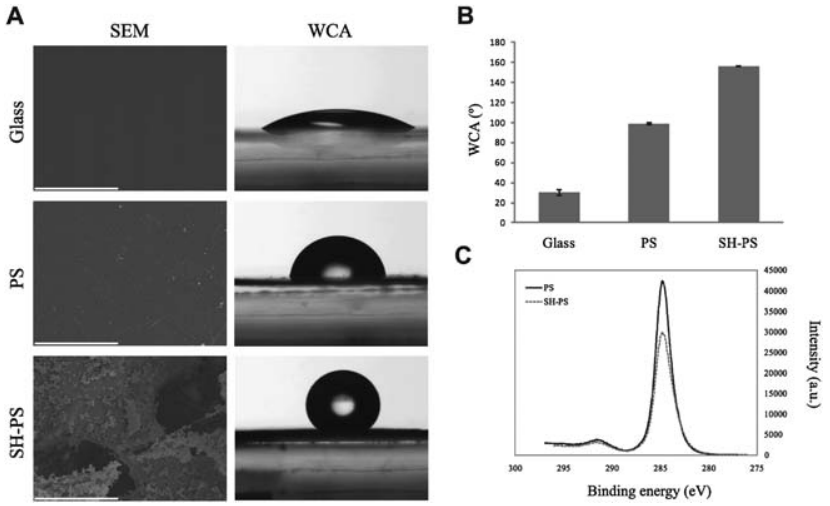

Fig. 1 Material surfaces. (A) SEM images of the different material surfaces: glass, polystyrene (PS) and superhydrophobic polystyrene (SHPS). A representative image of a drop of water on each one of the surfaces is shown. (B) Water contact angle (WCA) on the different surfaces as calculated from images in (A). Error bars represent the standard deviation of three independent experiments. (C) XPS spectra in the $\mathrm{C} 1 \mathrm{~s}$ regions for PS and SH-PS. Scale bar is $50 \mu \mathrm{m}$.

Table 1 Roughness parameters of smooth polystyrene (PS) and superhydrophobic-PS (SH-PS) measured by AFM. $R_{\mathrm{a}}$ is the arithmetic average of the height deviations from the center plane; $R_{\mathrm{ms}}$ is defined as the standard deviation of the height values; and $R_{\max }$ is the difference between the highest and lowest heights. The side of the square where the measures were conducted is also indicated

\begin{tabular}{llccc}
\hline & Size $/ \mu \mathrm{m}$ & $R_{\mathrm{ms}} / \mathrm{nm}$ & $R_{\mathrm{a}} / \mathrm{nm}$ & $R_{\max } / \mathrm{nm}$ \\
\hline PS & 2 & $1.44 \pm 0.07$ & $1.13 \pm 0.05$ & $10.24 \pm 1.30$ \\
SH-PS & 2 & $548 \pm 148$ & $395 \pm 157$ & $4291 \pm 213$ \\
\hline
\end{tabular}

the surface of the drop in contact with the substrate is suspended by air pockets. ${ }^{37}$ The relation between $\theta^{*}$, the WCA on the $\mathrm{SH}$ PS, and $\theta$, the WCA on the corresponding smooth PS, is

$$
\cos \theta^{*}=f(1+\cos \theta)-1
$$

where $f$ is the fraction of the liquid-solid contact. Taking for $\theta^{*}$ and $\theta$ the measured values in Fig. 1, the estimated $f$ is 0.1 which suggests that less than $10 \%$ of the drop will be in contact with the material surface. Even if the extrapolation cannot be directly done to the situation when the substrate is surrounded by the biological milieu, this fact will modify protein adsorption and cell behavior at the interface with the SH-PS.

The surface density of adsorbed FN was quantified by western blotting analyzing the amount of protein remaining in the supernatant after adsorption on the material surface. A calibration curve was built loading gels with known amounts of FN and the resulting bands were quantified by image analysis making use of the Otsu's algorithm to systematically identify the band borders. ${ }^{36}$ Each experiment included two reference points so that the position of the whole calibration curve could be checked each time. Fig. 2A shows the surface density of FN on PS, SH-PS and control glass after adsorption from a solution of concentration $20 \mu \mathrm{g} \mathrm{mL} \mathrm{m}^{-1}$. There is no statistical difference between the amount of adsorbed FN on PS and the control glass (approximately $450 \mathrm{ng} \mathrm{cm}^{-2}$ ). In contrast, the amount of FN on
A

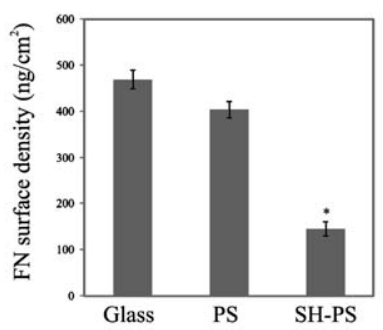

C

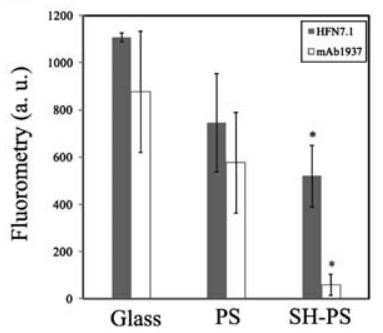

B

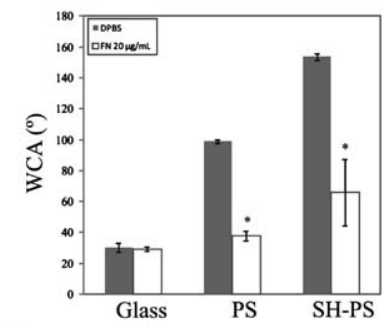

D

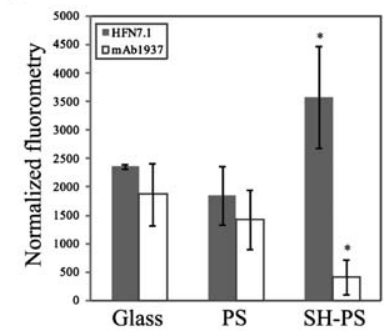

Fig. 2 Fibronectin adsorption on the different surfaces. (A) FN surface density after adsorption from a solution of concentration $20 \mu \mathrm{g} \mathrm{mL}^{-1}$. (B) Water contact angle on the different surfaces after FN adsorption from a solution of concentration $20 \mu \mathrm{g} \mathrm{mL}^{-1}$; as a control, the same experiment was performed with DPBS. (C) Monoclonal antibody binding for HFN7.1 and mAb1937 after FN adsorption from a solution of concentration $20 \mu \mathrm{g} \mathrm{mL}^{-1}$. (D) Monoclonal antibody binding for HFN7.1 and mAb1937 normalized to the surface density of adsorbed FN obtained in (A). Error bars represent the standard deviation of three independent experiments. ${ }^{*}$ stands for $p<0.05$.

the SH-PS is significantly lower, roughly $150 \mathrm{ng} \mathrm{cm}^{-2}$. These data were not corrected for the real surface area in the case of SH-PS. The roughness (Table 1) will considerably increase the surface area on SH-PS, which is counteracted by the fact that only part of the surface will actually be in contact with the material surface.

Protein adsorption on superhydrophobic surfaces is assumed to be more difficult than on more wettable ones, even if there is no precise quantification of the total amount adsorbed in the literature. ${ }^{7,38}$ That FN adsorption occurs indeed on the SH-PS was confirmed by measuring WCA after FN adsorption (Fig. 2B). WCA diminished in both PS and SH-PS as a consequence of the presence of $\mathrm{FN}$, which turns the surface into a more wettable one (as expected, wettability is not altered on the control glass after FN adsorption even if the surface is completely covered by the protein). ${ }^{39}$ As a proper control for the change in WCA after FN adsorption to PS and SH-PS, the exposure of the material surfaces to solutions without protein confirms that DPBS does not alter the wettability of the material per se (Fig. 2B).

Additionally, FN adsorption on SH-PS occurs in a different conformation as compared to PS and the control glass. The availability of the cell adhesion domains on the adsorbed FN was evaluated by ELISA with monoclonal antibodies, which is a well established method to probe for structural or conformational changes in adsorbed proteins. ${ }^{40,41}$ The antibody used (HFN7.1) was directed against the flexible linker between the $9^{\text {th }}$ and $10^{\text {th }}$ type III repeats of FN. ${ }^{42}$ The antibody mAb1937 is directed against the $8^{\text {th }}$ type III repeat of FN. It has been previously demonstrated that HFN7.1 is a receptor-mimetic probe for 
integrin binding and cell adhesion. ${ }^{43}$ Altogether both antibodies allow us to account for the availability of the RGD domain (located within the $10^{\text {th }}$ type III repeat of FN) and the PHSRN synergy sequence (located within the $9^{\text {th }}$ type III repeat of FN) respectively. ${ }^{43}$

Fig. 2C shows that the intensity of the ELISA signal for both HFN7.1 and mAb1937 is much lower for FN adsorbed on the superhydrophobic sample. That this is a direct consequence of the amount of adsorbed FN is better grasped if the fluorescence intensity is normalized to the previously measured FN surface density. Fig. 2D shows that even though less FN is adsorbed on the SH-PS, it displays the $9^{\text {th }}$ and $10^{\text {th }}$ type III repeats. Also, the comparison between the intensity level for each antibody on each surface shows that the same level is obtained for both HFN7.1 and mAb1937 on PS and the control glass but the intensity is much higher on the SH-PS for HFN7.1 as compared to mAb1937, which suggests that FN is not only adsorbed in a lower density on the SH-PS but also in a different conformation of the domains involved in cell adhesion, which suggests denaturation of the protein upon adsorption on the SH-PS. Since the availability of the RGD and synergy sequences is mandatory for cell adhesion to occur via the $\alpha_{5} \beta_{1}$ integrin, ${ }^{44-46}$ it is hypothesized that cell interaction on the superhydrophobic surfaces will involve other routes. This fact might constitute a potential risk for denaturation and coagulation of proteins in the biomedical applications of superhydrophobic surfaces in blood contacting devices. ${ }^{47,48}$

FN distribution at the microscale on the different material surfaces is observed in Fig. 3 by confocal microscopy after immunolabelling the adsorbed protein. A homogeneous layer of FN is extended on PS. In contrast, the rough surface morphology of the superhydrophobic sample, that is known to prevent liquid intrusion into its valleys, ${ }^{22,49}$ leads to uneven distribution of $\mathrm{FN}$ with micro-regions (in black) not covered by the protein.

Superhydrophobic surfaces were obtained by altering the surface roughness, as a combination of micro- and nano-metre scale roughness, along with a low surface energy material. ${ }^{32}$ It is well known that both surface micro- and nanotopography influence protein adsorption, including the amount of adsorbed protein and distribution between valleys and peaks. ${ }^{50-53}$ However, these modifications in the surface roughness did not involve extreme conditions of wettability, even if WCA was

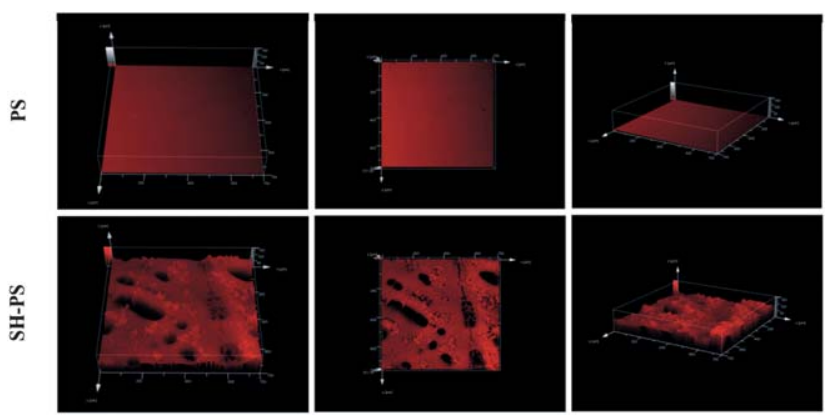

Fig. 3 Fibronectin distribution at the microscale on PS and SH-PS. 3D confocal images were performed after immunolabelling adsorbed FN from a solution of concentration $20 \mu \mathrm{g} \mathrm{mL}^{-1}$ on the different substrates. The size of the square is $750 \mu \mathrm{m}$, the vertical scale is $150 \mu \mathrm{m}$. altered as a consequence of topographic cues. Here, however, surface topography was altered in such a way that wettability is extremely modified, leading to qualitatively different phenomena at the material interface, such as the existence of areas on the material surface that are not in contact with the liquid and the appearance of specific forces that leads to denaturation of the adsorbed proteins. In this sense, it has been reported the formation of an immobile FN shell at liquid-air interfaces on highly hydrophobic PDMS micropillars, on which the FN solution remained suspended exhibiting a nearly spherical shape ${ }^{54}$ which agrees with the non-homogeneous adsorption of FN throughout the surface (Fig. 3).

\section{Cell adhesion and signaling on superhydrophobic-PS}

The formation of stable links between the extracellular matrix and the cell interior leads to integrin clustering and organization into focal adhesions. Fig. 4 shows the distribution of vinculin for cells on PS, SH-PS and the control glass after 3 hours of culture. Well-defined focal adhesions were found on both the control glass and PS, which also includes the development of the actin cytoskeleton. In contrast, vinculin is not organized into focal adhesions on the SH-PS, but randomly distributed throughout the cell. Accordingly, the actin cytoskeleton is not completely matured and mostly peripheral, which suggest the initial states of polymerization of F-actin. ${ }^{55}$ Similar results are shown when other focal adhesion proteins are followed after adhesion on the different surfaces, as it is shown for both talin and paxillin (Fig. S1 $\dagger$ ). The situation is not altered after one day of culture as it is shown for cells in Fig. S $2 \dagger$.

The cell morphology was also drastically altered on the SH-PS as compared to either PS or the control glass. ${ }^{13}$ Fig. 5 shows the
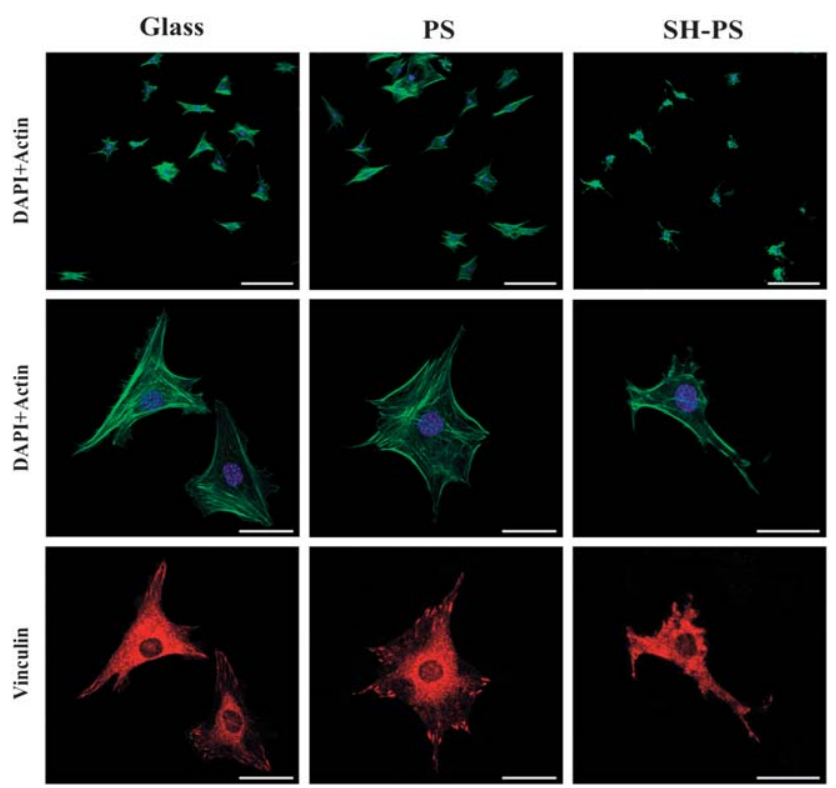

Fig. 4 Cytoskeleton organization and focal adhesion formation. Cells were maintained for 3 hours on the different FN-coated (from a solution of $20 \mu \mathrm{g} \mathrm{mL}^{-1}$ ) surfaces. First and second rows show F-actin cytoskeleton at different magnifications (scale bar 150 and $30 \mu \mathrm{m}$ respectively). The third one shows the distribution of the focal adhesion protein vinculin (scale bar $30 \mu \mathrm{m}$ ). Nuclei were counterstained with DAPI. 
cell morphology as observed by SEM. Cells are well spread and with multiple filopodia on PS and the control glass. In contrast, cells on the SH-PS present several morphologies, which include rounded morphologies and extended ones. Extended cells adhere to the SH-PS surface through cytoplasmatic projections, which has been suggested to be a consequence that the FN solution is not in contact with the entire material surface giving rise to areas uncovered by the protein (Fig. 3). ${ }^{21}$ Likewise, this is related to the effect of surface micro/nanotopography on cell behavior. The effect of surface topography on cell adhesion has been widely investigated. It is well-known that surface microtopography promotes changes in cell adhesion patterns, cell orientation, and cell shape on the substrate. ${ }^{56}$ Cells cultured on smooth surfaces tend to generate more organized extracellular matrix (ECM), including more homogeneous distribution of focal adhesions. However, on rougher surfaces, focal adhesions are located at cell edges, where the contact with the substrate takes place. ${ }^{57}$ This fact can also modify the cell phenotype and influence cell morphology and differentiation. ${ }^{58,59}$

Within the cell-protein-material interaction paradigm, though cells react to topographic cues, it is not because they do interact directly with the surface of the synthetic material - whatever its microtopography - but as a consequence of the ECM proteins somehow immobilized onto the substrate. As explained previously, FN is adsorbed in lower quantity and altered conformation and distribution (Fig. 2) on SH-PS, which we argued to be a consequence of extreme surface wettability rather than a direct effect of the surface roughness. Likewise, the formation of focal adhesions and cell morphology is triggered by $\mathrm{FN}$ adsorption: diminished FN surface density in altered conformation (Fig. 2) led to lack of focal adhesion formation and actin organization (Fig. 4 and $\mathrm{S} 1 \dagger$ ).

Focal adhesion kinase (FAK) localizes to focal adhesions to activate multiple signaling pathways that regulate cell migration, survival, proliferation, and differentiation..$^{60-64} \mathrm{We}$ examined the phosphorylation of tyrosine-397, the autophosphorylation site in FAK and a binding site for src and PI-3 kinase ${ }^{65,66}$ by western
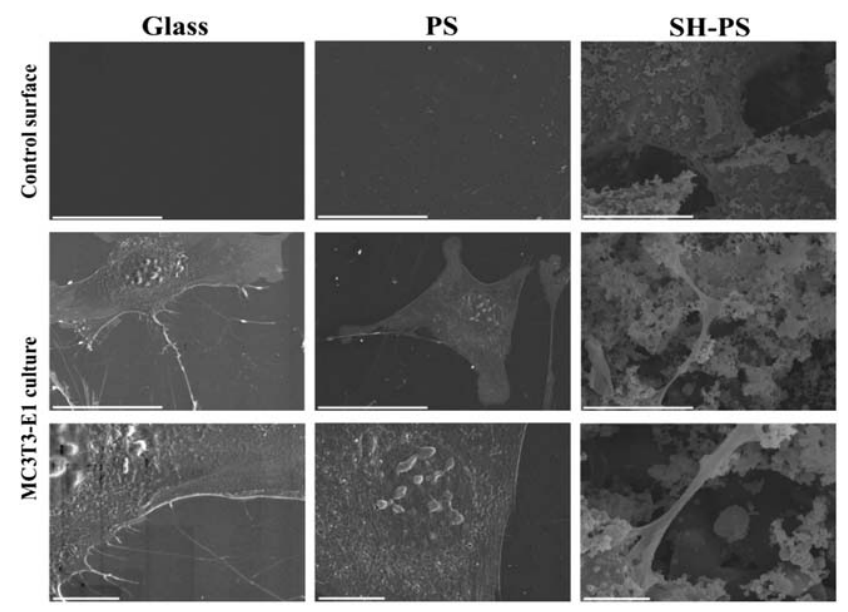

Fig. 5 Cell morphology as observed by SEM. Cells were cultured for $3 \mathrm{~h}$ on the different FN-coated surfaces. Well-spread cells are observed on PS and the control glass, with multiple filopodia. Altered cell morphology is obtained on SH-PS. The scale bar is 10,10 and $50 \mu \mathrm{m}$ for each row respectively. blot (Fig. 6). Although the band for FAKs remains approximately constant, the level of phosphorylation (pFAKs) is much lower for cells on the SH-PS (Fig. 6A). Likewise, the ratio between phosphorylated and total FAKs is much lower on the SH-PS than PS and glass (Fig. 6B). Surface wettability is known to direct FAK phosphorylation, which was enhanced on hydrophilic rather than hydrophobic substrates. ${ }^{67}$ On the side, the fact that FAK phosphorylation on the control glass (hydrophilic) and PS (hydrophobic) occurs in the same way is one more evidence to support that surface wettability is a rough parameter to account for the effect of surface properties on protein adsorption and cell interaction. ${ }^{6}$

\section{Matrix reorganization and cell contractility on superhydrophobic-PS}

After initial adhesion on FN-coated materials, cells attempt to reorganize the adsorbed layer of proteins at the material interface. ${ }^{26}$ Thus, cells reorganize their environment continuously before matrix secretion starts. This evidence raises the possibility that the biological compatibility of materials might be connected with the ability of cells to remodel surface associated proteins presumably as an attempt to form their own matrix, e.g. materials that bind proteins loosely will support the organization of a provisional extracellular matrix. ${ }^{30}$

Fig. 7 shows the cellular reorganization of adsorbed $\mathrm{FN}$ after 2.5 and $5 \mathrm{~h}$ of culture on the different surfaces, including the control glass. It is observed that cells are able to reorganize FN on the control glass as shown by the dark area nearby the cell. Reorganization occurs much less actively on PS: some movements of the adsorbed FN layer take place on this surface, but the dark areas in the pericellular zone are smaller and mostly coincident with focal adhesion plaques. In contrast,
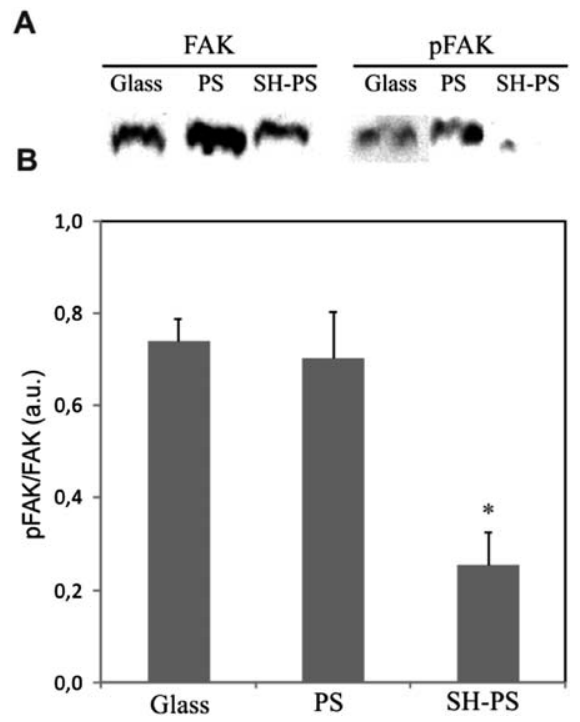

Fig. 6 Phosphorylation of FAKs for cells on FN-coated substrates. (A) Representative western blot for total and phosphorylated tyrosine-397 residue on FAK (FAK and pFAK respectively). (B) Quantification of the fraction of phosphorylated FAKs by image analysis of the western blot bands. Error bars represent the standard deviation of three independent experiments. ${ }^{*}$ stands for $p<0.05$. 


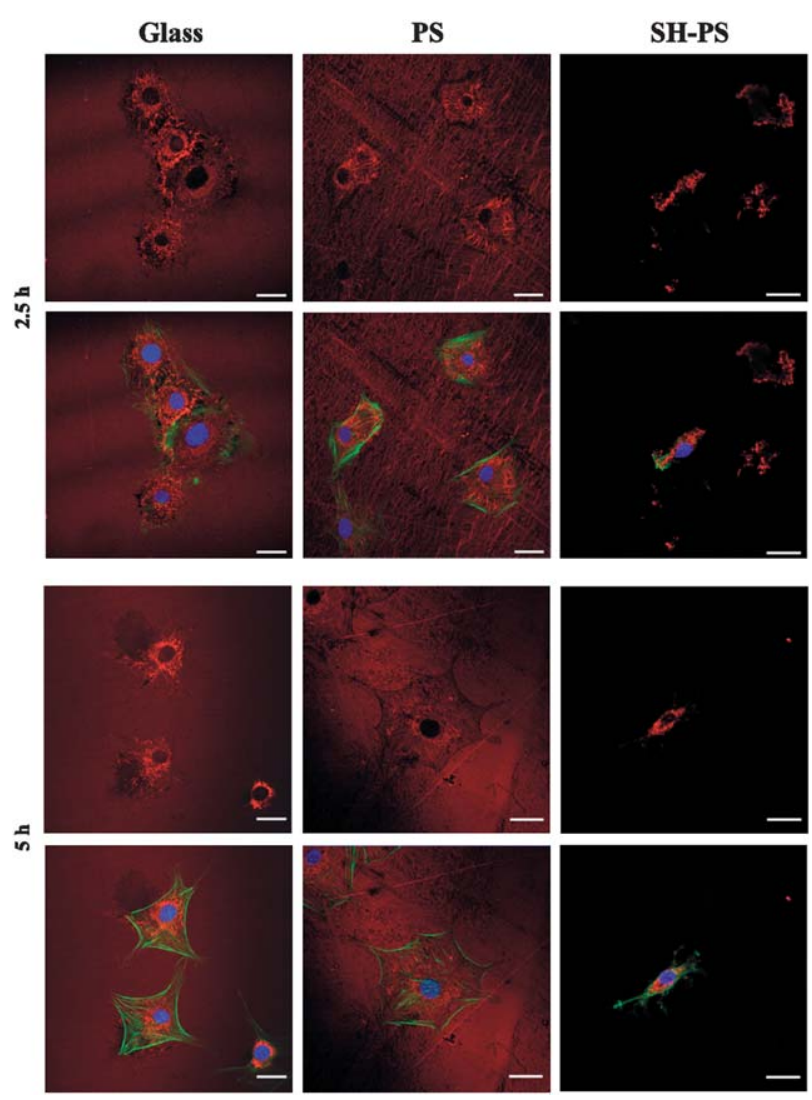

Fig. 7 Cellular reorganization of adsorbed $\mathrm{FN}$ on the different substrates after 2.5 and $5 \mathrm{~h}$ of culture on the different substrates. Actin cytoskeleton is included in the bottom images for the sake of identification of cells. Nuclei were counterstained with DAPI. Scale bar is $30 \mu \mathrm{m}$.

reorganization is completely absent on the SH-PS, and mostly the initially adsorbed FN is observed in the areas on which cells are attached. It must be commented here that the intensity of the fluorescence for the FN layer cannot be used to estimate the amount of adsorbed protein, since the laser parameters and image processing were not uniform between samples but adjusted to get better identification of the reorganization phenomena in each case.

Cell contractility results from dynamic interactions between actin filaments and myosin, which are regulated via phosphorylation of myosin light chain (MLC). Rho GTPases control the formation of stress fibers and focal adhesion assembly by modulating MLC phosphorylation and generating actin-myosin contractility ${ }^{68}$ After activation, Rho acts through its effector Rho-kinase (ROCK) to enhance the contraction of cells by either inactivation of myosin phosphatase or phosphorylation of MLC. Rho/ROCK signaling was hyperactive in the absence of FAK, as reflected by increased MLC phosphorylation. ${ }^{69}$ Rho activity can result in elevated stress fiber formation and stabilization of focal adhesions. ${ }^{70}$ Interestingly, inhibition of MLC-driven contractility did not alter integrin binding (bound density and distribution) to the ECM but it resulted in the dissolution of focal adhesions as indicated by reduced localization of vinculin and talin to adhesion structures. Additionally, it has been demonstrated that FAK phosphorylation mediated the effects of MLC phosphorylation on serum-dependent cell adhesion strengthening. The formation of mature focal adhesions and FAK phosphorylation have been correlated with cell contractility and strengthening. ${ }^{69}$

We obtained the same FAK phosphorylation levels on both glass and PS (Fig. 6), as well as similar patterns for MLC phosphorylation (Fig. 8), which suggest similar ability of cells to exert mechanical strength on the underlying surface. Since reorganization occurs much more actively on the control glass than PS (Fig. 7) one must conclude that the strength of interaction between FN and the substrate is higher on PS than on the control glass. And the other way around, since adhesion occurs without the formation of mature focal adhesions (Fig. 4), lower levels of FAK phosphorylation (Fig. 6) and the absence of MLC phosphorylation (Fig. 8) on SH-PS, cells are not able to actively reorganize the underlying $\mathrm{FN}$ adsorbed on the superhydrophobic substrate (Fig. 7).

Late FN matrix formation, after different time periods, is shown in Fig. S3†. The secretion and organization of FN fibrils are enhanced on PS and the control glass, i.e. on the substrates on which FN reorganization takes place more intensively. Scarce FN formation is obtained on the superhydrophobic substrate, and only the initially adsorbed FN is observed on the images after 3 and 5 days of culture. This fact suggests that late matrix formation is in need not only of cell adhesion on the substrate, but some cell movements, in the range of the size of the focal adhesion plaques, must also take place so matrix deposition takes place normally. ${ }^{50}$ Late matrix formation has been related to the ability of cells to rearrange the initially adsorbed protein layer, especially when comparing cell adhesion on hydrophilic and hydrophobic substrates. ${ }^{71}$

Overall, the mechanistic model proposed by Kato and Mkrsich $^{72}$ can be used to correlate the physico-chemical properties of the superhydrophobic surfaces to fibronectin adsorption and cell adhesion. This model proposed that each polymerization event of cell receptors could be considered to be a consequence of either a nucleation or a growth mechanism. Nucleation refers to
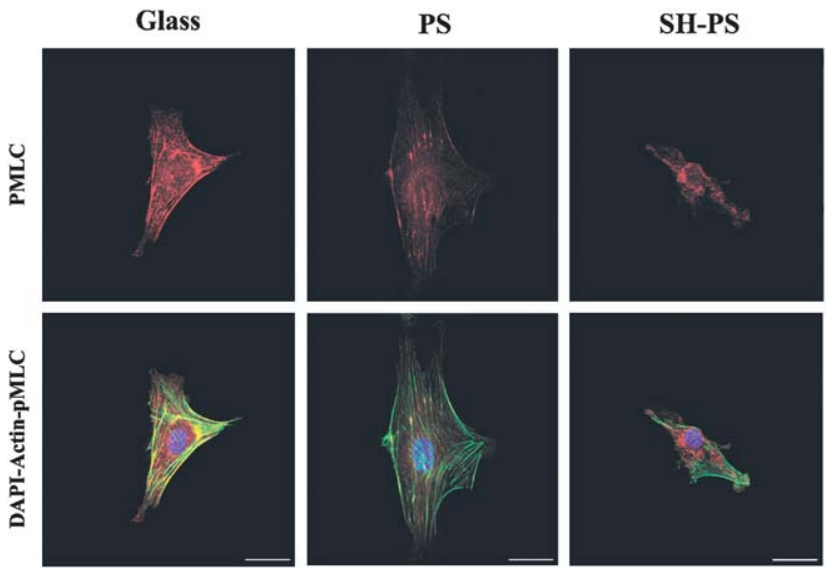

Fig. 8 Phosphorylation of myosin light chain (pMLC) observed by fluorescence staining for cells cultured on the different FN-coated substrates. The superposition of pMLC and actin cytoskeleton is shown in the bottom row. Nuclei were counterstained with DAPI. Scale bar is 30 $\mu \mathrm{m}$. 
the aggregation of some integrin receptors into a cluster that does not dissociate (nucleation of focal adhesions). This cluster becomes irreversible because an intracellular network of proteins cross-links the integrin receptors together. On the other hand, growth describes the subsequent process of individual receptors (and smaller, mobile clusters) diffusing to the nucleated clusters (growth of focal adhesions). SH-PS presents the same surface chemistry as smooth PS (Fig. 1C), but the surface roughness is modified such that superhydrophobicity occurs and the full contact between the liquid and the material surfaces is prevented (Table 1). As a consequence, hydrophobic forces appeared at the material interface, ${ }^{73}$ which perturbs water structure and leads to FN adsorption in altered conformation (the synergy domain is less available, Fig. 2D). ${ }^{74}$ On SH-PS, the minimum spatial density of adsorbed $\mathrm{FN}$ for focal adhesions to be developed is not reached. In contrast, higher number of focal contacts was formed on smooth PS (and the control glass) distributed throughout the cytoplasm (favored nucleation). Afterwards, cell-signaling (phosphorylation of FAKs), cell contractility and matrix reorganization do not occur on the SH-PS, neither does FN secretion.

\section{Long term studies}

The stability of the cell population on the superhydrophobic surfaces was followed up to 21 days of culture. Cell density was calculated on PS, SH-PS and the control glass and it is shown in Fig. 9. Cells are able to adhere and proliferate on every surface, although cell density is significantly lower on the SH-PS surface. In other studies the detrimental effect of superhydrophobic surfaces on cell adhesion and proliferation was more evident. Cell density of bone marrow derived cells on superhydrophobic PLLA decreased monotonically from day 1 to day 7 of culture, and virtually no cells could be found after 21 days of culture, ${ }^{21}$ no platelet could adhere on nanostructured superhydrophobic surfaces ${ }^{5}$ and only some proliferation of fibroblasts was found on a superhydrophobic plasma polymer after 3 days. ${ }^{22}$ The results indicate that there is no universal behavior of cells on

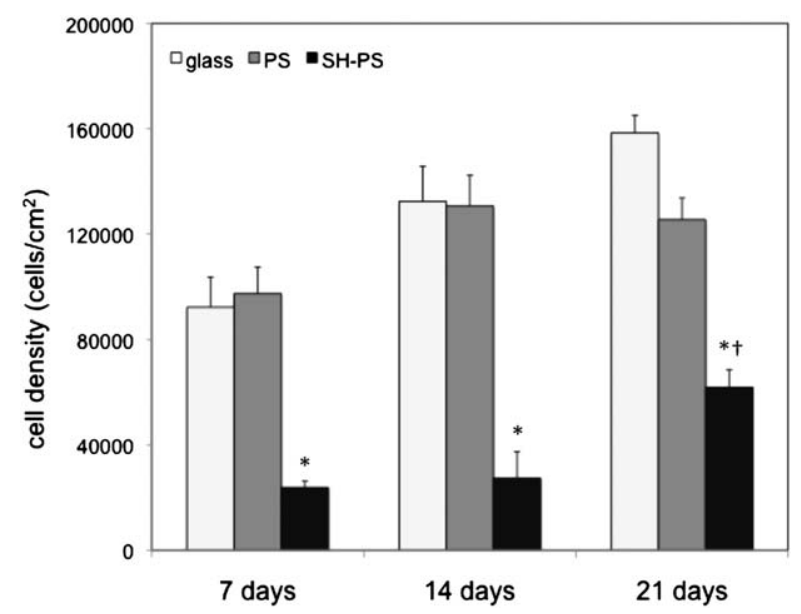

Fig. 9 Cell density on the different surfaces (glass, PS, and SH-PS) after 7, 14 and 21 days of culture. Error bars represent the standard deviation of three independent experiments. (* stands for $p<0.05$ for each culture time, $\uparrow$ stands for $p<0.05$ in SH-PS after 21 days of culture). superhydrophobic surfaces, and dependencies should exist on the nature of the surface, its specific topography and on the cell type.

\section{Conclusions}

We have shown that FN is adsorbed on superhydrophobic PS surfaces in lower density and altered conformation as compared with the corresponding standard PS surface. As a consequence, cell adhesion occurs without formation of mature focal adhesion plaques and scarce phosphorylation of FAKs. Under these circumstances, neither cell contractility nor reorganization of adsorbed FN nor secretion of newly synthesized FN fibrils occurs on the SH-PS. In contrast, cells are able to proliferate and maintain a stable population up to 21 days on the superhydrophobic substrate, although in a significantly lower density when compared with standard PS.

\section{Acknowledgements}

The support of the Spanish Ministry of Science and Innovation through project MAT2009-14440-C02-01 is acknowledged. CIBER-BBN is an initiative funded by the VI National R\&D\&i Plan 2008-2011, Iniciativa Ingenio 2010, Consolider Program, CIBER Actions and financed by the Instituto de Salud Carlos III with assistance from the European Regional Development Fund. This work was supported by funds for research in the field of Regenerative Medicine through the collaboration agreement from the Conselleria de Sanidad (Generalitat Valenciana), and the Instituto de Salud Carlos III.

\section{Notes and references}

1 A. Michiardi, C. Aparicio, B. D. Ratner, J. A. Planell and J. Gil, Biomaterials, 2007, 28, 586-594.

2 Y. Arima and H. Iwata, Biomaterials, 2007, 28, 3074-3082.

3 J. Y. Lim, M. C. Shaughnessy, Z. Zhou, H. Noh, E. A. Vogler and H. J. Donahue, Biomaterials, 2008, 29, 1776-1784.

4 K. L. Prime and G. M. Whitesides, J. Am. Chem. Soc., 1993, 115, 10714-10721.

5 T. Sun, H. Tan, D. Han, Q. Fu and L. Jiang, Small, 2005, 1, 959-963.

6 D. Gugutkov, G. Altankov, J. C. Rodríguez Hernández, M. Monleón Pradas and M. Salmerón-Sánchez, J. Biomed. Mater. Res., Part A, 2010, 92, 322-331.

7 H. J. Lee and S. J. Michielsen, Polym. Sci. Pol. Phys., 2007, 45, $253-$ 261.

8 Y. I. Yoon, H. S. Moon, W. S. Lyoo, T. S. Lee and W. H. Park, J. Colloid Interface Sci., 2008, 320, 91-95.

9 Z. Yuan, H. Chen, J. Tang, X. Chen, D. Zhao and Z. Wang, Surf. Coat. Technol., 2007, 201, 7138-7142.

10 J. Fresnais, J. P. Chapel and F. Poncin-Epaillard, Surf. Coat. Technol., 2006, 200, 5296-5305.

11 B. Geiger, A. Bershadsky, R. Pankov and K. M. Yamada, Nat. Rev. Mol. Cell Biol., 2001, 2, 793-805.

12 Y. Lee, S. H. Park, K. B. Kim and J. K. Lee, Adv. Mater., 2007, 19, 2330-2335.

13 T. Sun, L. Feng, X. Gao and L. Jiang, Acc. Chem. Res., 2005, 38, 644 652.

14 Y. Zhu, J. Zhang, Y. Zheng, Z. Huang, L. Feng and L. Jiang, $A d v$. Funct. Mater., 2006, 16, 568-574.

15 S. Wang, L. Feng and L. Jiang, Adv. Mater., 2006, 18, 767-770.

16 R. Langer and D. A. Tirrell, Nature, 2004, 428, 487-492.

17 N. Peppas and R. Langer, Science, 1994, 263, 1715-1720.

18 R. Banerjee, K. Nageswari and R. R. Puniyani, J. Biomater. Appl., $1997,12,57-76$.

19 B. W. Song, D. D. Veiga, C. A. Custódio and J. F. Mano, Adv. Mater., 2009, 21, 1830-1834.

20 T. Sun, H. Tan, D. Han, Q. Fu and L. Jiang, Small, 2005, 1, 959-963. 
21 N. M. Alves, J. Shi, E. Oramas, J. L. Santos, H. Tomás and J. F. Mano, J. Biomed. Mater. Res., Part A, 2009, 91, 480-488.

22 T. Ishizaki, N. Saito and O. Takai, Langmuir, 2010, 26, 8147-8154.

23 K. Anselme, Biomaterials, 2000, 21, 667-681.

24 R. O. Hynes, Cell, 2002, 110, 673-687.

25 R. O. Hynes, Cell, 1987, 48, 549-554.

26 E. D. Hay, Cell Biology of Extracellular Matrix, Springer, 2nd edn, 1991, p. 468.

27 F. Grinnell, J. Cell Biol., 1986, 103, 2697-2706.

28 Z. Avnur and B. Geiger, Cell, 1981, 1, 121-132.

29 R. Christopher, A. Kowalczyk and P. McKeown-Longo, J. Cell Sci., 1997, 110, 569-581.

30 G. Altankov, F. Grinnell and T. Groth, J. Biomed. Mater. Res., 1996, 30, 385-391.

31 N. M. Oliveira, A. I. Neto, W. Song and J. F. Mano, Appl. Phys. Express, 2010, 3, 085205.

32 X. Feng and L. Jiang, Adv. Mater., 2006, 18, 3063-3078.

33 H. Y. Erbil, A. L. Demirel, Y. Avci and O. Mert, Science, 2003, 299, $1377-1380$

34 J. Shi, N. M. Alves and J. F. Mano, Bioinspiration Biomimetics, 2008, 3, 034003.

35 S. M. Oliveira, W. Song, N. M. Alves and J. F. Mano, Soft Matter, 2011, DOI: $10.1039 / \mathrm{c} 1 \mathrm{sm} 05943 \mathrm{~b}$, in press.

36 P. Rico, J. C. Rodríguez Hernández, D. Moratal, G. Altankov, M. Monleón Pradas and M. Salmerón-Sánchez, Tissue Eng., Part A, 2009, 15, 3271-3281.

37 B. D. Cassie and S. Baxter, Physics, 1944, 546-551.

38 X. Yao, Y. Song and L. Jiang, Adv. Mater., 2011, 23, 719-734.

39 H. Matsumura, K. Kawasaki and M. Kambara, Colloids Surf., B, 1997, 8, 181-188.

40 T. P. Ugarova, C. Zamarron, Y. Veklich, R. D. Bowditch, M. H. Ginsberg, J. W. Weisel and E. F. Plow, Biochemistry, 1995, 34, 4457-4466.

41 K. B. McClary, T. Ugarova and D. W. Grainger, J. Biomed. Mater. Res., 2000, 50, 428-439.

42 R. C. Schoen, K. L. Bentley and R. J. Klebe, Hybridoma (19812001), 1982, 1, 99-108.

43 B. G. Keselowsky, D. M. Collard and A. J. García, J. Biomed. Mater. Res., Part A, 2003, 66, 247-259.

44 R. Pankov and K. M. Yamada, J. Cell Sci., 2002, 115, 3861-3863.

45 S. Aota, M. Nomizu and K. Yamada, J. Biol. Chem., 1994, 269, 24756-24761.

46 S. D. Redick, J. Cell Biol., 2000, 149, 521-527.

47 C. Mao, Y. Qiu, H. Sang, H. Mei, A. Zhu, J. Shen and S. Lin, Adv. Colloid Interface Sci., 2004, 110, 5-17.

48 J. A. Chinn, T. A. Horbett and B. D. Ratner, Thromb. Haemostasis, 1991, 65, 608-617.

49 A. J. García, J. E. Schwarzbauer and D. Boettiger, Biochemistry, 2002, 41, 9063-9069.
50 C. González-García, S. Sousa, D. Moratal, P. Rico and M. SalmerónSánchez, Colloids Surf., B, 2010, 77, 181-190.

51 E. Costa Martínez, J. C. Rodríguez Hernández, M. Machado, J. F. Mano, J. L. Gómez Ribelles, M. Monleón Pradas and M. Salmerón Sánchez, Tissue Eng., Part A, 2008, 14, 1751-1762.

52 P. E. Scopelliti, A. Borgonovo, M. Indrieri, L. Giorgetti, G. Bongiorno, R. Carbone, A. Podestà and P. Milani, PLoS One, 2010, 5, e11862.

53 M. Pegueroles, C. Aparicio, M. Bosio, E. Engel, F. Gil, J. A. Planell and G. Altankov, Acta Biomater., 2010, 6, 291-301.

54 J. Ulmer, B. Geiger and J. P. Spatz, Soft Matter, 2008, 4, 1998-2007.

55 M. D. Welch and R. D. Mullins, Annu. Rev. Cell Dev. Biol., 2002, 18, $247-288$.

56 A. Curtis and C. Wilkinson, Biomaterials, 1997, 18, 1573-1583.

57 K. Anselme, M. Bigerelle, B. Noel, E. Dufresne, D. Judas, A. Iost and P. Hardouin, J. Biomed. Mater. Res., 2000, 49, 155-166.

58 O. Zinger, G. Zhao, Z. Schwartz, J. Simpson, M. Wieland, D. Landlolt and B. Boyan, Biomaterials, 2005, 26, 1837-1847.

59 M. J. Dalby, N. Gadegaard, R. Tare, A. Andar, M. O. Riehle, P. Herzyk, C. D. W. Wilkinson and R. O. C. Oreffo, Nat. Mater., 2007, 6, 997-1003.

60 D. Ilić, Y. Furuta, S. Kanazawa, N. Takeda, K. Sobue, N. Nakatsuji, S. Nomura, J. Fujimoto, M. Okada and T. Yamamoto, Nature, 1995, 377, 539-544.

61 L. A. Cary, J. F. Chang and J. L. Guan, J. Cell Sci., 1996, 109, 1787-1794.

62 S. M. Frisch, K. Vuori, E. Ruoslahti and P. Y. Chan-Hui, J. Cell Biol., 1996, 134, 793-799.

63 J. H. Zhao, H. Reiske and J. L. Guan, J. Cell Biol., 1998, 143, 19972008.

64 V. J. Thannickal, D. Y. Lee, E. S. White, Z. Cui, J. M. Larios, R. Chacon, J. C. Horowitz, R. M. Day and P. E. Thomas, J. Biol. Chem., 2003, 278, 12384-12389.

65 M. D. Schaller, J. D. Hildebrand, J. D. Shannon, J. W. Fox, R. R. Vines and J. T. Parsons, Mol. Cell. Biol., 1994, 14, 1680-1688.

66 H. R. Reiske, S. C. Kao, L. A. Cary, J. L. Guan, J. F. Lai and H. C. Chen, J. Biol. Chem., 1999, 274, 12361-12366.

67 B. G. Keselowsky, D. M. Collard and A. J. García, Biomaterials, 2004, 25, 5947-5954.

68 B. Geiger, A. Bershadsky, R. Pankov and K. M. Yamada, Nat. Rev. Mol. Cell Biol., 2001, 2, 793-805.

69 K. E. Michael, D. W. Dumbauld, K. L. Burns, S. K. Hanks and A. J. García, Mol. Biol. Cell, 2009, 20, 2508-2519.

70 D. W. Dumbauld, K. E. Michael, S. K. Hanks and A. J. García, Biol. Cell, 2010, 102, 203-213.

71 G. Altankov, T. Groth, N. Krasteva, W. Albrecht and D. Paul, J. Biomater. Sci., Polym. Ed., 1997, 8, 721-740.

72 M. Kato and M. Mrksich, Biochemistry, 2004, 43, 2699-2707.

73 D. Chandler, Nature, 2005, 437, 640-647.

74 L. Lins and R. Brasseur, FASEB J., 1995, 9, 535-540. 